\title{
Foveal lesions do not ameliorate the visual discrimination deficit that follows foveal prestriate lesions
}

\author{
ROBERT BAKER, KENNETH L. KLOTHEN, and CHARLES G. GROSS \\ Princeton University, Princeton, New Jersey 08540
}

\begin{abstract}
Removal of foveal prestriate cortex impairs visual discrimination learning by rhesus monkeys. To test whether this deficit might be due to a "higher-order" foveal scotoma, monkeys with foveal prestriate lesions were forced to fixate extrafoveally by infliction of central retinal lesions. The retinal lesions did not improve their visual discrimination learning, thereby failing to support the hypothesis.
\end{abstract}

In the monkey, lesions of foveal prestriate cortex produce a severe impairment in visual discrimination learning (see reviews: Gross, 1973a, b). Foveal prestriate cortex includes ventrolateral prestriate cortex and extends to the posterior border of cytoarchitectonic area $\mathrm{TE}$, thereby including a portion of posterior temporal cortex (see Figure 1). More anterior lesions of the inferior temporal convexity that are confined to cytoarchitectonic area TE [and termed by Cowey \& Gross (1970) "inferotemporal lesions"] also impair visual discrimination learning, but the two impairments are different from each other in several ways. Relative to inferotemporal lesions, foveal prestriate lesions produce (a) greater impairment on discrimination of two-dimensional patterns and of three-dimensional objects, (b) less sensitivity to reinforcement contingencies, and (c) less impairment in a concurrent learning paradigm (see reviews: Gross, 1973a, b).

The foveal prestriate deficit appears to be particularly marked on visual problems that depend heavily on the use of foveal information (Gross, 1973b). Anatomical and electrophysiological evidence also suggests that foveal prestriate cortex is specialized for the processing of foveal information. This area receives direct and indirect projections from lateral striate cortex, which represents about the central $6 \mathrm{deg}$ of the visual field (Cowey, 1973; Zeki, 1969, 1971). Furthermore, single neurons in at least the anterior portions of foveal prestriate cortex have receptive fields that invariably include the fovea (Gross, Rocha-Miranda, \& Bender, 1972).

Ablation of lateral striate cortex or destruction of the macula itself produces relatively little difficulty on visual discrimination tasks, particularly if the tasks do not

We would like to thank Dr. R. Carr for making the retinal lesions, Mr. W. Nyberg and Professor A. Laties for retinal photography, and Ms. C. Curcio for cortical histology. Supported by NIH Grant MH-19420 and NSF Grant 27612x. Robert Baker is now at College of Physicians and Surgeons, Columbia University. Reprint requests should be sent to $C$. $G$. Gross, Department of Psychology, Green Hall, Princeton University, Princeton, New Jersey 08540.

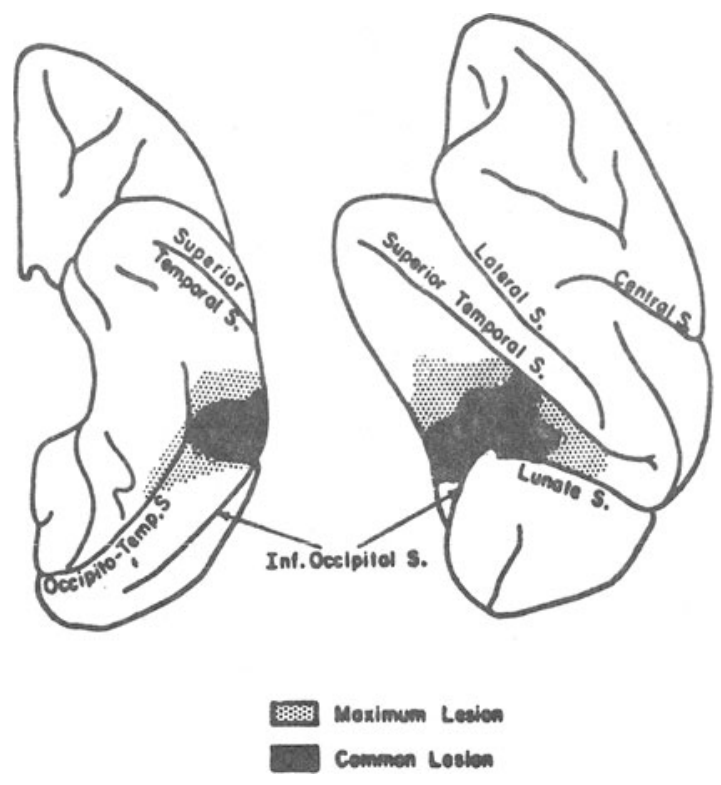

Figure 1. Ventral (left) and lateral view of left cerebral hemisphere showing extent of foveal prestriate lesions in a previous study. The lesions in the left hemisphere have been transferred to the right. Reconstructions, cross sections and thalamic degeneration of the individual lesions on which this composite is based have been published (Cowey \& Gross, 1970). The lesions in the present study were very similar to the illustrated "common lesion" and fell within the bounds of the illustrated "maximum lesion."

demand fine acuity (e.g., Cowey \& Weiskrantz, 1967; Wilson \& Mishkin, 1959). Presumably this small discrimination deficit is because monkeys with either lesion learn to fixate extrafoveally, substituting the extrafoveal portions of striate and prestriate cortex for the foveal portions (Cowey, 1967). If, by contrast, after foveal prestriate lesions, monkeys continued to fixate foveally, then the critical information for learning visual discriminations could not be processed in cortex beyond striate cortex, since the cortical targets of foveal striate cortex are missing. That is, the foveal prestriate lesions 
may produce a "higher-order" foveal scotoma. If foveal prestriate lesions did produce such an effect, then forcing monkeys with foveal prestriate lesions to fixate extrafoveally should eliminate or ameliorate their deficit on visual discrimination tasks (Cowey \& Gross, 1970).

In the present investigation, we tested the hypothesis that monkeys with foveal prestriate lesions are impaired in visual discrimination because of a "higher-order" foveal scotoma by destroying the macula of such animals and thereby producing extrafoveal fixation. According to the hypothesis, monkeys with combined lesions of foveal prestriate cortex and the fovea should perform similarly to normal monkeys or, at least, better than monkeys with lesions of foveal prestriate cortex and normal retinae.

\section{METHODS}

\section{Subjects and surgical procedures}

Ten immature Macaca mulatta weighing between 1.9 and $4 \mathrm{~kg}$ at the start of the experiment were used as subjects. Six animals received bilateral lesions of foveal prestriate cortex (Nos. 1-6), and four did not receive any cortical lesions (Nos. 7-10). Three animals with cortical lesions (Nos. 4-6) and one unoperated animal (No.7) subsequently received binocular lesions of the macular portion of the retina. The animals with only cortical lesions (Nos. 1-3) are known as the cortex only group, the animals with both cortical and retinal lesions (Nos. 4-6) as the cortex plus retina group, and the remaining animals (Nos. 7-10) as the unoperated group.

The foveal prestriate lesions were intended to be similar to those previously made (e.g., Cowey \& Gross, 1970), i.e., to extend rostrally to about $10 \mathrm{~mm}$ in front of the ascending limb of the inferior occipital sulcus, caudally to the anterior bank of the lunate sulcus, dorsally to the depth of the inferior bank of the superior temporal sulcus, and ventrally to the lateral bank of the occipito-temporal sulcus and to include both banks of the ascending inferior occipital sulcus anterior to the lunate sulcus and the medial bank of this sulcus for about $4 \mathrm{~mm}$ posterior to the lunate sulcus (see Figure 1). They corresponded approximately to the " $0+$ I" lesions of Iwai and Mishkin (1969). The cortical surgery was performed aseptically under Nembutal anesthesia.

The retinal lesions were produced under Nembutal anesthesia (after topical administration of a $1 \%$ solution of tropicamide) with either an argon arc photocoagulator or a ruby laser.

\section{Apparatus}

The animals were trained in a sound insulated box. Two translucent Gerbrands keys $1-1 / 8$ in. $(2.9 \mathrm{~cm})$ in diam and 12 in. $(30.5 \mathrm{~cm})$ apart were mounted in one wall of the box. The discriminanda were back projected onto the keys by rear-projection readout units (series 10 Industrial Electronic Engineers, Inc.) modified so that the keys were in the plane of the image of the stimulus. A liquid dispenser was mounted in the center of the same wall 12 in. $(30.5 \mathrm{~cm})$ below a line connecting the two keys. The interior of the box was illuminated from above, and a masking noise was present. Stimulus presentation and reinforcement were programmed automatically. This apparatus was the same as that previously used by Cowey and Gross (1970).

\section{Behavioral Procedures}

The monkeys were water deprived and tested for 100 trials per day, 7 days each week. On all tasks, the spatial position of the stimuli was randomized. Responses during the intertrial interval reset the interval. Response to either or both keys turned the stimuli off. A correct response was followed by presentation of $.25 \mathrm{cc}$ orange juice. Correction trials were not used.

Preliminary training. The animals were first trained to respond to the keys when illuminated with a white square (Grason-Stadler stimulus 156-4), and then were trained to discriminate the white square (positive) from an unlighted blank key to a criterion of 45 correct responses in 50 trials.

First discrimination. The animals were trained to discriminate two horizontal white lines (positive) from two vertical ones (Grason-Stadler 154-5,6) to a criterion of 90 correct responses in 100 trials on each of 2 successive days. Six animals then received their foveal prestriate lesions. About 14 days later, all the animals were retrained to the original criterion, or, if they failed to attain it, for a maximum of 1,500 trials. About a month later, four animals received their retinal lesions, and all animals then were retrained for a third time on the discrimination to the original criterion or for a maximum of 1,500 trials.

Second discrimination. The animals were trained to discriminate a triangle from its mirror image (Grason-Stadler $150-1,2)$ to a criterion of 90 correct responses in 100 trials on 2 successive days or for a maximum of 1,500 trials.

Third discrimination. The animals were trained to discriminate an $\mathrm{E}$ from an I (both rotated $90 \mathrm{deg}$ counterclockwise) to a criterion of 90 correct responses in 100 trials on 2 successive days or for a maximum of 1,500 trials.

\section{Summary of Design}

The order of events was as follows: (1) Preliminary training. (2) First discrimination task. (3) Foveal prestriate cortex lesion (cortex only and cortex plus retina groups). (4) Retention of first discrimination task. (5) Retinal lesion (cortex plus retina group and Animal 7). (6) Retention of first discrimination task (again). (7) Second discrimination task. (8) Third discrimination task.

\section{Determination of Retinal Lesions}

The retinae were photographed with a fundus camera. In addition, the size and location of the lesions were plotted with an ophthalmoscope when the monkeys were anesthetized, immobilized with gallamine triethiodide, and artificially respirated. The borders of the retinal lesion and of the blind spot were plotted on a tangent screen using a corner-cube mirror placed in the path of the ophthalmoscope beam. Histological examination of the retina of one animal indicated destruction of the ganglion cell layer. Presumably similar destruction had occurred in the other cases as well.

All the retinal lesions included the fovea, were roughly circular, and had a mean area of 24.5 square degrees. There was no correlation between the size or location of the retinal lesions and performance on the behavioral tasks.

\section{Histological Procedures and Resalts}

At the end of the experiment, the monkeys were anesthetized with Nembutai and perfused through the dorsal aorta with $.9 \%$ saline and then $10 \%$ Formalin. The head was placed in a stereotaxic machine and the dorsal skull and dura removed. A cut was made in front of and behind the lesion in stereotaxic vertical planes, and a steel pin was passed horizontally through each hemisphere, making a small hole which provided a baseline for reconstructing the brain from serial coronal sections. The brain was then removed, placed in $10 \%$ Formalin for about 7 days followed by sucrose Formalin (10\% Formalin, 30\% sucrose) until it sank, and then frozen sections were cut 25 microns thick. Every 10 th section was stained with cresyl violet. Reconstructions of the lesions were made from sections $1 \mathrm{~mm}$ apart, with an examination of additional intermediate sections when necessary.

Examination of the brains prior to sectioning suggested that 
Table 1

Errors to Criterion

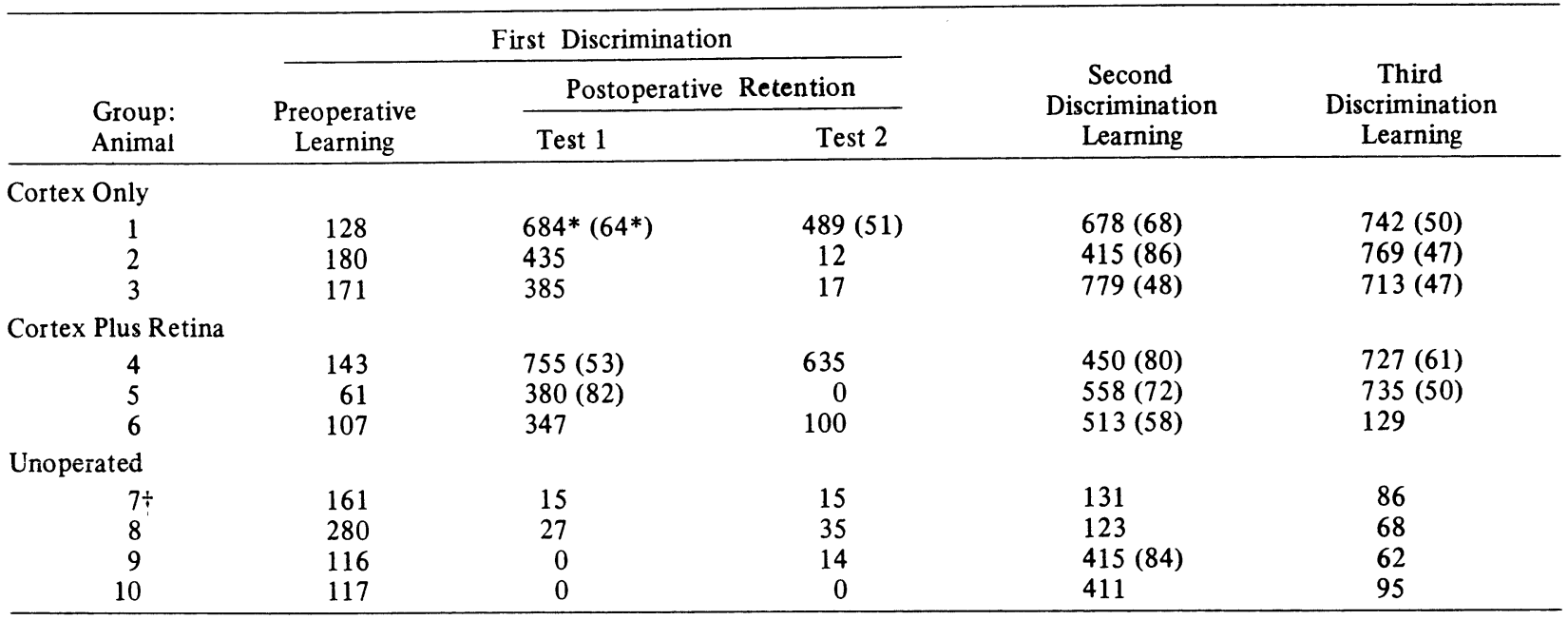

*In the case of animals failing to reach criterion in 1,500 trials, the total number of errors and (in parentheses) the percentage correct in the final 100 trials are given.

tAnimal 7 had a retinal but no cortical lesion.

all had lesions similar to each other and similar to the foveal prestriate lesions in our previous studies (see Figure 1). Full histological processing and lesion reconstruction was carried out for only three animals; the results confirmed the close similarity of the lesions in these animals to our previous foveal prestriate lesions (e.g., Cowey \& Gross, 1970). There was no correlation between variation in the lesions and variation on the behavioral tasks.

\section{RESULTS}

\section{First Discrimination}

Table 1 indicates the number of errors each animal made prior to reaching criterion on each task or, if the animal failed to reach criterion, the total number of errors and the per cent correct in the final 100 trials. Since, throughout the study, the performance of the one animal with only a retinal lesion (No. 7) was very similar to that of the unoperated animals, its data were pooled with those of the unoperated group.

Prior to operation, the three groups made a similar number of errors in learning to discriminate the horizontal and vertical lines. After surgery, the animals with foveal prestriate lesions were very significantly impaired in retention of this discrimination relative to the normal animals as measured by errors to criterion $(\mathrm{U}=0, \mathrm{p}=.01$, Mann-Whitney $\mathrm{U}$ test $)$. On this first retention test, the performance of the animals with foveal prestriate lesions that were to receive retinal lesions did not differ significantly from the performance of those that were not $(U=2)$. One animal in each of these groups (Nos. 1 and 4) was still performing near chance at the completion of the allotted maximum number of trials. Another animal in the cortex plus retina group (No.5), although also failing to reach criterion, was performing over $80 \%$ correct at the end of training.
On the second retention test, the unoperated group showed excellent retention as did the two animals in the cortex only group that had previously relearned the problem. The one cortex only animal (No.1) that had previously failed to relearn the problem continued to fail. After their retinal lesions, the animal that had previously reached criterion (No.6) and the animal that was performing over $80 \%$ correct (No.5) reattained criterion, but the animal that had previously performed near chance continued to do so (No.4). Statistically, none of the groups differed from each other.

In summary, the results of the first retention test indicated that in every case, the foveal prestriate lesions produced the expected severe discrimination deficit. The second retention test did not provide an adequate test of the hypothesis because, prior to the retinal lesion, one of the cortex plus retina group had already learned the problem, and a second had almost done so. However, the failure of the third cortex plus retina animal to improve after its retinal lesion argued against a reduction in the deficit after retinal lesions.

\section{Second Discrimination}

As expected, the performance of the normal animals was far superior to that of the operated ones $(U=1$; $p=.02$ ). However, there were no significant differences between the cortex only and cortex plus retina groups $(U=3)$. The unoperated animals leamed this discrimination in a mean of 360 errors, but after the allotted 1,500 trials, no operated animal had reached criterion, although one animal in each group was performing at least $80 \%$ correct.

\section{Third Discrimination}

Again the performance of the normal animals was superior to that of the operated ones $(U=0, p=.01)$, 
and again the operated groups did not differ significantly from each other $(U=2)$. Whereas all the normal animals reached criterion within 95 errors, no operated animal except for one in the cortex plus retina group reached criterion in the allotted 1,500 trials. This one exception (No. 6) who learned after 129 errors had also been the best performer among the animals with cortical lesions on the initial retention test before its retinal lesion.

In summary, the results of the second and third discrimination tasks failed to show any amelioration of the foveal prestriate deficit following the addition of foveal retinal lesions.

\section{DISCUSSION}

The purpose of this experiment was to test the idea that animals with foveal prestriate lesions are impaired on visual pattern discrimination tasks because of a "higher-order" foveal scotoma. According to this hypothesis, forcing extrafoveal fixation by inflicting foveal lesions should have eliminated or at least ameliorated the deficit produced by removal of foveal prestriate cortex; extrafoveal fixation would have enabled the information critical for visual discrimination to be normally processed at the level of prestriate cortex.

The results were unambiguous: all the animals with foveal prestriate lesions were severely impaired on visual discrimination tasks, and the addition of foveal lesions gave no indication of reducing this impairment. Thus, there was no support for the hypothesis that animals with foveal prestriate lesions have a higher-order foveal scotoma. However, since the retinotopic organization of foveal prestriate cortex is still not known in detail, it is possible that the retinal lesions were too small to shift fixation sufficiently to insure that information from the new fixation point was processed in intact prestriate cortex.

\section{REFERENCES}

Cowey, A. Perimetric study of field defects in monkeys after cortical and retinal ablations. Quarterly Journal of Experimental Psy chology, 1967, 19, 232-245.

Cowey, A. Brain damage and seeing: A new look at some old problems. Transactions of the Ophthalmological Societies of the United Kingdom, 1973, 93, 409-416.

Cowey, A., \& Gross, C. G. Effects of foveal prestriate and inferotemporal lesions on visual discriminations by rhesus monkeys. Experimental Brain Research, 1970, 11, 128-144.

Cowey, A. \& Weiskrantz, L. A comparison of the effects of inferotemporal and striate cortex lesions on the visual behavior of rhesus monkeys. Quartedy Journal of Experimental Psy chology, 1967, 19, 246-253.

Gross, C. G. Visual functions of inferotemporal cortex. R. Jung (Ed.), Handbook of sensory physiology (Vol. 7/3B). Berlin and New York: Springer-Verlag, 1973. (a)

Gross, C. G. Inferotemporal cortex and vision. Progress in Phy siological Psy chology, 1973, 5, 77-123. (b)

Gross, C. G., Rocha-Miranda, C. E., \& Bender, D. B. Visual properties of neurons in inferotemporal cortex of the macaque. Journal of Neurophysiology, 1972, 35, 96-111.

Iwai, E., \& Mishkin, M. Further evidence on the locus of the visual area in the temporal lobe of the monkey. Experimental Neurology, 1969, 25, 585-594.

Wilson, W. A., Jr., \& Mishkin, M. Comparison of the effects of inferotemporal and lateral occipital lesions on visually guided behavior in monkeys. Joumal of Comparative and Phy siological Psychology, 1959, 52, 10-17.

Zeki, S. M. Representation of central visual fields in prestriate cortex of monkey. Brain Research, 1969, 14, 271-291.

Zeki, S. M. Cortical projections from two prestriate areas in the monkey. Brain Research, 1971, 34, 19-35.

(Received for publication February 13, 1975; revision accepted March 7,1975 .) 